\title{
ON RESTRICTED UNIQUENESS FOR SYSTEMS OF ORDINARY DIFFERENTIAL EQUATIONS
}

\author{
J. M. BOWNDS ${ }^{1}$ AND J. B. DIAZ
}

\begin{abstract}
A uniqueness theorem is proved, for not necessarily Lipschitzian systems of ordinary differential equations, $y^{\prime}=f$. This theorem compares with one of Okamura and Murakami, in that, here, at the expense of assuming a certain additional smoothness for $f$ on open sets, no assumption is made regarding the existence of an auxiliary positive definite (Lyapunov) function. An example compares the relative applicability of the two theorems.
\end{abstract}

Let $I$ denote the open real interval $(a, b)$, and let $O$ be an open set in real $n$-dimensional Euclidean space $E_{n}$. Suppose $f: \bar{I} \times \bar{O} \rightarrow E_{n}$ is continuous. Consider the initial value problem

$$
\begin{aligned}
y^{\prime}(x) & =f(x, y(x)), \quad \text { for all } x \in I, \\
y(a) & =y_{a} \in \bar{O} .
\end{aligned}
$$

By a solution is meant a continuous function $u: \bar{I} \rightarrow \bar{O}$, which is differentiable, and satisfies (1), on $I$, and also satisfies (2). Clearly, if $u$ is a solution, and $f$ is continuous on $\bar{I} \times \bar{O}$, then the derivative $u^{\prime}$, by (1), will be continuous on $I$.

The main concern of this paper is to prove a theorem, which will be compared with the results of Okamura [1] and Murakami [2] (see, also, Lakshmikantham and Leela [3]), which are based upon the existence of a certain positive-definite auxiliary (Lyapunov) function; see [2] or [3] for the actual statement. These results, stated in [2] and [3], are valid for the infinite dimensional initial value problem; to emphasize the simplicity of the present approach, however, only the finite dimensional case is considered here. The present theorem also compares to a result of Brauer and Sternberg [4], who obtain local uniqueness by assuming, as in [1] and [2], the existence of a positive-definite, Lyapunov-type auxiliary function.

Received by the editors April 29, 1972 and, in revised form, June 2, 1972.

AMS (MOS) subject classifications (1970). Primary 34A10, 34A40; Secondary 34A01, 34A02.

Key words and phrases. Ordinary differential equations, initial-value problems, uniqueness, Lyapunov functions.

1 The work of this author was supported by the Albert Einstein Chair of Science at Rensselaer Polytechnic Institute.

(c) American Mathematical Society 1973 
As pointed out by Murakami, uniqueness theorems which impose Lipschitz type inequalities on $f$ may be inapplicable in some cases where the Lyapunov function, referred to above, may actually be constructed. In particular, he notices that the right-hand member of the scalar problem:

$$
\begin{aligned}
y^{\prime}(x)=f(x, y) & =1+\sqrt{ } y, & & y \geqq 0, \\
& =1, & & y<0,
\end{aligned}
$$

cannot satisfy the condition

$$
\langle f(x, y)-f(x, z), y-z\rangle \leqq M\|y-z\|^{2},
$$

which is required, for example, in the results of Browder [5] and Kato [6] (in the scalar case, this condition reduces to

$$
\left.(f(x, y)-f(x, z))(y-z) \leqq M(y-z)^{2}\right) ;
$$

however, it is shown that the required Lyapunov function exists, by displaying the complicated expression:

$$
\begin{array}{rlrl}
\Phi(x, y, z) & & \\
& =[\sqrt{ } y-\sqrt{ } z-\log (1+\sqrt{ } y)+\log (1+\sqrt{ } z)]^{2}, & & y \geqq 0, \quad z \geqq 0, \\
(5) & =\left[\sqrt{ } y-\log (1+\sqrt{ } y)-\frac{1}{2} z\right]^{2}, & & y \geqq 0, \quad z<0, \\
& =\left[\frac{1}{2} y-\sqrt{ } z+\log (1+\sqrt{ } z)\right]^{2}, & & y<0, \quad z \geqq 0, \\
& =\frac{1}{4}[y-z]^{2}, & & y<0, \quad z<0 .
\end{array}
$$

Hence, the initial value problem (3), (4) has a unique solution. Note here that this particular construction is based on a specific knowledge of the solution to (3), (4), which is given, in implicit form, by $\sqrt{ } y-\log (1+\sqrt{ } y)=$ $\frac{1}{2} x$. It may be pointed out here that this uniqueness also follows as a trivial corollary to the theorem in [7], in which the proof is motivated by one of Fine [8]; see also Hartman [9, Theorem 8.1, p. 109].

In view of the complication of (5), it appears that, for even slightly more involved equations than (3), for example, $y^{\prime}=\sqrt{ } y+x^{2}$, there may be great difficulty in determining the required Lyapunov function (our attention was drawn, to this particular example, by Professor V. Lakshmikantham). The following theorem offers an alternative approach, which can be applied to many equations which do not satisfy a Lipschitz inequality, and which do not admit an obvious $\Phi$ in the Murakami result. In effect, the Murakami positive-definite, continuously differentiable function $\Phi(x, y, z)$ is replaced, simply and explicitly, by $V(x, y, z)=$ $\frac{1}{2}\|f(x, y)-f(x, z)\|^{2}$, which may be merely positive semidefinite. Of 
course, in this way, the need for any kind of construction of a suitable function $\Phi(x, y, z)$, as in (5), is absent.

With the above notation, assume $f \in C^{1}(I \times O)$, and define $F: I \times O \rightarrow E_{n+1}$ and $\phi: I \times O \rightarrow E_{n}$, by the equations $F(x, y)=(1, f(x, y))$, and $\phi(x, y)=$ $\left(\left\langle\nabla f_{1}, F\right\rangle,\left\langle\nabla f_{2}, F\right\rangle, \cdots,\left\langle\nabla f_{n}, F\right\rangle\right)$, where $f=\left(f_{1}, \cdots, f_{n}\right), \nabla$ denotes the gradient in $E_{n+1}$, and $\langle$,$\rangle is the scalar product, in E_{n+1}$.

THEOREM. If $\langle f(x, y)-f(x, z), \phi(x, y)-\phi(x, z)\rangle \leqq 0$, for all $(x, y)$, $(x, z) \in I \times O$, where $\langle$,$\rangle is the sca!ar product in E_{n}$, then there exists at most one solution $u$ to (1), (2) on $I$, such that $u(x) \in O$ for $x \in I$.

It is to be noted that the inequality in the hypothesis, when written in terms of the given real-valued functions $f_{1}, f_{2}, \cdots, f_{n}$, is just

$$
\begin{aligned}
\sum_{i=1}^{n}\left[f_{i}(x, y)-f_{i}(x, z)\right][ & \left(\frac{\partial f_{i}}{\partial x}(x, y)+\sum_{j=1}^{n} \frac{\partial f_{i}}{\partial y_{j}}(x, y) f_{j}(x, y)\right) \\
& \left.-\left(\frac{\partial f_{i}}{\partial x}(x, z)+\sum_{j=1}^{n} \frac{\partial f_{i}}{\partial z_{j}}(x, z) f_{j}(x, z)\right)\right] \leqq 0,
\end{aligned}
$$

where $y=\left(y_{1}, y_{2}, \cdots, y_{n}\right), z=\left(z_{1}, z_{2}, \cdots, z_{n}\right)$, and, where, using the customary notation, $\partial f_{i} / \partial y_{j}$ and $\partial f_{i} / \partial z_{j}$ denote the same partial derivative.

Before the proof, some remarks are in order. First, depending upon the choice of the open set $O$, there may be no solution satisfying $u(x) \in O$, for $x \in I$, or there may exist one or many solutions for which $u(x) \in O$, for $x \in I$. For example, if $n=1, a=0, b=1, y_{0}=0, y^{\prime}=(|y|)^{1 / 2}$, and

$$
I \times O=\left\{(x, y): 0<x<1, \frac{1}{4}<y<1\right\},
$$

then there is no solution in $O$. On the other hand, if

$$
I \times O=\{(x, y): 0<x<1,0<y<1\},
$$

then $u(x)=\frac{1}{4} x^{2}$ for $0 \leqq x \leqq 1$ gives, as predicted by the theorem, the only solution for which $u(x) \in O$ for $0<x<1$. Finally, the main point here is that $f$ may fail to satisfy a Lipschitz condition, due to its behavior near the boundary of $I \times O$; clearly, if the initial point $y_{a}$ is an interior point of $O$, with all the other conditions being satisfied, then there is a locally unique solution, owing to the fact that the continuous differentiability of $f$ then implies a Lipschitz condition in a suitably chosen neighborhood of $\left(a, y_{a}\right)$.

Proof. Let $\|\cdot\|$ denote the Euclidean norm in $E_{n}$, and define

$$
V(x)=\frac{1}{2}\|f(x, u(x))-f(x, v(x))\|^{2},
$$

for $a \leqq x \leqq b$, where $u$ is a solution, and $v$ is a solution, in the sense of the theorem. Then, $V(a)=0, V(x) \geqq 0$ for $a \leqq x \leqq b$, and the derivative $V^{\prime}(x)$ 
is given by

$$
V^{\prime}(x)=\langle f(x, u(x))-f(x, v(x)),(d / d x)[f(x, u(x))-f(x, v(x))]\rangle,
$$

for $x \in I=(a, b)$. But, using the above definitions of $F$ and $\phi$, and the "chain rule" of the differential calculus, it follows that

$$
(d / d x)[f(x, u(x))-f(x, v(x))]=\phi(x, u(x))-\phi(x, v(x)) .
$$

Hence, the hypothesis of the theorem then implies that $V^{\prime}(x) \leqq 0$ for $x \in I$. Thus, it must be true that $V(x) \equiv 0$ on $\bar{I}$; that is, $f(x, u(x)) \equiv$ $f(x, v(x))$ on $I$. But, then, the differential equations satisfied, on $I$, by $u$ and $v$, imply that $u^{\prime}(x)=v^{\prime}(x)$ for all $x \in I$, while the initial condition gives that $u(a)=v(a)$; therefore, $u(x)=v(x)$ for all $x \in I$. Q.E.D.

As a simple example, consider the scalar problem

$$
\begin{aligned}
& y^{\prime}=f(x, y)=\sqrt{ } y+g(x), \quad y \geqq 0,0<x<1, \\
& y(0)=0, \\
& =g(x), \quad y<0,0<x<1,
\end{aligned}
$$

where $g \in C[0,1], C^{\prime}(0,1)$, and $g(x) \geqq 0$ for $x \geqq 0$. Then, with $I=(0,1)$, and $I \times O=\{(x, y) ; x \in I$ and $y>0\}$, one has, on $I \times O$ :

$$
\phi(x, y)=\langle\nabla f,(1, f)\rangle=g^{\prime}(x)+\frac{1}{2} y^{-1 / 2}\left[y^{1 / 2}+g(x)\right] .
$$

Hence, for $(x, y),(x, z) \in I \times O$,

$$
\begin{gathered}
\langle f(x, y)-f(x, z), \phi(x, y)-\phi(x, z)\rangle=\frac{1}{2} g(x)\left[y^{1 / 2}-z^{1 / 2}\right]\left[y^{-1 / 2}-z^{-1 / 2}\right] \\
=-\frac{1}{2} g(x) y^{-1 / 2} z^{-1 / 2}\left[y^{1 / 2}-z^{1 / 2}\right]^{2} \leqq 0
\end{gathered}
$$

as required by the hypothesis of the theorem. The conclusion is that there is at most one solution $u(x)$ for which $u(x)>0$ when $x>0$. If $g(x)>0$ for $x>0$, then any local solution must be positive for $x>0$; hence the local solution (given by Peano's theorem) must be the only positive solution in its local domain of definition.

\section{REFERENCES}

1. H. Okamura, Condition nécessaire et suffisante remplie par les équations différentielles ordinaires sans points de Peano, Mem. Coll. Sci. Kyoto Imp. Univ. Ser. A. 24 (1942), 21-28. MR 7, 442.

2. H. Murakami, On non-linear ordinary and evolution equations, Funkcial. Ekvac. 9 (1966), 151-162. MR 35 \#514.

3. V. Lakshmikantham and S. Leela, Differential and integral inequalities. Vol. II, Academic Press, New York, 1969.

4. F. Brauer and S. Sternberg, Local uniqueness, existence in the large, and the convergence of successive approximations, Amer. J. Math. 80 (1958), 421-430. MR 20 \#1806. 
5. F. Browder, Non-linear equations of evolution, Ann. of Math. (2) 80 (1964), 485523. MR 30 \#4167.

6. T. Kato, Integration of the equation of evolution in a Banach space, J. Math. Soc. Japan 5 (1953), 208-234. MR 15, 437.

7. J. Bownds, $A$ uniqueness theorem for $y^{\prime}=f(x, y)$ using a certain factorization of $f$, J. Differential Equations 7 (1970), 227-231.

8. A. W. Hales, G. R. Sells and N. J. Fine, Multiple solutions of a differential equation (Problems and solutions), Amer. Math. Monthly 73 (1966), 672.

9. P. Hartman, Ordinary differential equations, Wiley, New York, 1964. MR 30 \#1270.

Department of Mathematics, University of Arizona, Tucson, Arizona 85721

Department of Mathematics, Rensselaer Polytechnic Instrtute, Troy, New YORK 12181 